PROCEEDINGS OF THE

AMERICAN MATHEMATICAL SOCIETY

Volume 130, Number 3, Pages 613-618

S 0002-9939(01)06393-6

Article electronically published on October 23, 2001

\title{
ON THE NATANZON-TURAEV COMPACTIFICATION OF THE HURWITZ SPACE
}

\author{
STEVEN P. DIAZ
}

(Communicated by Michael Stillman)

\begin{abstract}
Natanzon and Turaev have constructed by topological methods a compactification of the Hurwitz space, that is, the space of simple branched covers of the two-sphere. Here we show that this compactification is homeomorphic to a compactification mentioned by Diaz and Edidin (in 1996) that was constructed by algebraic methods. Using this we are able to show by example that the Natanzon-Turaev compactification can be singular, that is, not a manifold.
\end{abstract}

\section{INTRODUCTION}

The Hurwitz space $H^{n, w}$ is the set of all $n$ sheeted connected coverings of the sphere $S^{2}$ simply branched over exactly $w$ distinct points and otherwise unbranched. Hurwitz $\left[\mathrm{Hu}\right.$ showed a natural way to make $H^{n, w}$ into a complex manifold of complex dimension $w$. The space $H^{n, w}$ is not compact. When two or more distinct branch points approach each other the limit of the corresponding covers will not be a cover of the same type. Various compactifications of $H^{n, w}$ and closely related spaces have been constructed and studied by methods of algebraic geometry; see for instance $[\mathrm{HM}],[\overline{\mathrm{DE}}]$, and $[\mathrm{M}]$. In $[\mathrm{NT}]$ Natanzon and Turaev construct a compactification of $H^{n, w}$ using topological methods. In this paper we show that the compactification constructed in [NT] is homeomorphic to a compactification in DE.

This allows us to answer some questions brought up in [NT. Natanzon and Turaev point out that there are no known topological descriptions of the compactifications in algebraic geometry. In view of the homeomorphism we construct, the Natanzon-Turaev compactification is a topological description of one of the compactifications from algebraic geometry. Finally, Natanzon and Turaev asked about the local structure of their compactification, in particular, is it a complex manifold? Methods of algebraic geometry allow one to analyse the local structure of the compactification from $[\mathrm{DE}$ to which the Natanzon-Turaev compactification is homeomorphic. Using this we construct an example to show that the NatanzonTuraev compactification can be singular, that is, not a complex manifold.

Received by the editors August 17, 1999 and, in revised form, March 8, 2000.

2000 Mathematics Subject Classification. Primary 14H10; Secondary 57M12.

Key words and phrases. Hurwitz space.

(C)2001 American Mathematical Society 


\section{THE HOMEOMORPHISM}

We start by describing the Natanzon-Turaev compactification and the compactification from $\mathrm{DE}$ to which it is homeomorphic.

First we describe the Natanzon-Turaev compactification. From the RiemannHurwitz formula one deduces that the genus of the cover of $S^{2}$ in an element of $H^{n, w}$ is $g=\frac{1}{2}(w-2 n+2)$. Fix a closed oriented connected (though [NT] does not require connected) surface $\Sigma$ of genus $g$. Define $H(\Sigma, n)$ to be the set of equivalence classes of $n$-sheeted simple branched coverings $f: \Sigma \rightarrow S^{2}$, where the equivalence relation is: $f: \Sigma \rightarrow S^{2}$ and $f^{\prime}: \Sigma \rightarrow S^{2}$ are equivalent if and only if there is a homeomorphism $\alpha: \Sigma \rightarrow \Sigma$ such that $f=f^{\prime} \alpha$. $H(\Sigma, n)$ is the Hurwitz space $H^{n, w}$. Natanzon and Turaev construct a compactification $N(\Sigma, n)$ of $H(\Sigma, n)$. The points of $N(\Sigma, n)$ are equivalence classes of decorated functions, where Natanzon and Turaev define decorated functions and their equivalence as follows. We quote directly from [NT].

Definition 2.1. A decorated function (on the surface $\Sigma$ ) is a triple $\left(f, E,\left\{D_{e}\right\}_{e \in E}\right)$ where $f: \Sigma \rightarrow S^{2}$ is a simple branched covering, $E$ is a finite subset of $S^{2}$ disjoint from the set of branch points of $f$, and $\left\{D_{e}\right\}_{e \in E}$ are disjoint closed 2-discs embedded in $S^{2}$ such that: $e \in \operatorname{Int} D_{e}$ for all $e \in E$, each $D_{e}$ contains at least two branch points of $f$, and the circles $\left\{\partial D_{e}\right\}_{e}$ do not contain branch points of $f$.

An isotopy of a decorated function $\left(f, E,\left\{D_{e}\right\}_{e}\right)$ is a family of decorated functions $\left(\varphi_{t} f, E,\left\{\varphi_{t}\left(D_{e}\right)\right\}_{e}\right)$ where $\left\{\varphi_{t}: S^{2} \rightarrow S^{2}\right\}_{t \in[0,1]}$ is an isotopy of the identity map $\varphi_{0}=\operatorname{id}_{S^{2}}$ such that for all $t \in[0,1]$ the homeomorphism $\varphi_{t}$ preserves (pointwise) $E$ and the branch points of $f$ lying outside $\bigcup_{e} D_{e}$.

We say that two decorated functions $\left(f, E,\left\{D_{e}\right\}_{e \in E}\right)$ and $\left(f^{\prime}, E^{\prime},\left\{D_{e}^{\prime}\right\}_{e \in E^{\prime}}\right)$ are equivalent if $E=E^{\prime}$ and $f^{\prime}$ may be obtained from $f$ by an isotopy and/or composition with a homeomorphism $\Sigma \rightarrow \Sigma$. (The isotopy must also take each $D_{e}$ to $\left.D_{e}^{\prime}.\right)$

Recall that the set of unordered sets of $k$ not necessarily distinct points in complex projective one-space $\mathbb{C P}^{1}=S^{2}$ is naturally identified with complex projective $k$-space $\mathbb{C P}^{k}=\mathbb{P}^{k}$. There is a natural map $q: H(\Sigma, n)=H^{n, w} \rightarrow \mathbb{P}^{w}$ which sends a cover $\left(f: \Sigma \rightarrow S^{2}\right) \in H(\Sigma, n)$ to its branch points. This extends to a map (also denoted $q) q: N(\Sigma, n) \rightarrow \mathbb{P}^{w}$ which sends a decorated function $\left(f, E,\left\{D_{e}\right\}\right)$ to the set consisting of the branch points of $f$ outside of $\bigcup_{e} D_{e}$ each counted once, plus the points of $E$ each counted with multiplicity equal to the number of branch points of $f$ inside the corresponding $D_{e}$. As mentioned in [NT] before Lemma 2.2 the extended mapping $q$ is continuous and open.

Now we describe the algebraic compactification mentioned in $[\mathrm{DE}]$. In that article the authors denote the compactification by $\overline{S H}_{k, b}(k$ corresponds to $n$ and $b$ to $w$ in $\left.H^{n, w}\right)$. It has been known since Hurwitz that $q: H(\Sigma, n) \rightarrow \mathbb{P}^{w}$ is finite. Therefore the function field of $H(\Sigma, n)$ is a finite extension of the function field of $\mathbb{P}^{w}$. The compactification $\overline{S H}_{k, b}$ is defined to be the normalization of $\mathbb{P}^{w}$ in the function field of $H(\Sigma, n)$. The map $q: H(\Sigma, n) \rightarrow \mathbb{P}^{w}$ extends to a regular algebraic morphism $\pi: \overline{S H}_{k, b} \rightarrow \mathbb{P}^{w}$.

The homeomorphism $g: N(\Sigma, n) \rightarrow \overline{S H}_{k, b}$ that we claim to exist will be defined to be the identity on $H(\Sigma, n) \subset N(\Sigma, n)$ and $H(\Sigma, n) \subset \overline{S H}_{k, b}$. It will also commute with the maps $q$ and $\pi$ to $\mathbb{P}^{w}$. What is left to define are the values of $g$ on the points of $N(\Sigma, n)$ lying over points of $\mathbb{P}^{w}$ corresponding to nondistinct points in $\mathbb{P}^{1}$. 
Denote by $D$ the points of $\mathbb{P}^{w}$ corresponding to nondistinct sets of $w$ points in $\mathbb{P}^{1}$. Let $p \in D$. Lemma 4.3 of $\left[\mathrm{DE}\right.$, which was proved for $\bar{H}_{k, b}$ can in the same way be proven for $\overline{S H}_{k, b}$ to obtain the following.

Lemma 2.1. Pick a small connected neighborhood (say any small open ball) $B$ of $p$ in $\mathbb{P}^{w}$, and pick a point $r \in B-D$. The fundamental group of $B-D$ with base point $r$ acts via monodromy on $\pi^{-1}(r)$. Define an equivalence relation on $\pi^{-1}(r)$ by saying that two points are equivalent if and only if they can be taken to each other by the monodromy action. For B sufficiently small the following are true.

1. Two points of $\pi^{-1}(r)$ lie in the same monodromy equivalence class iff they lie in the same connected component of $\pi^{-1}(B-D)$.

2. The closure of each connected component of $\pi^{-1}(B-D)$ in $\pi^{-1}(B)$ has exactly one point over $p$.

3. The closures of the connected components of $\pi^{-1}(B-D)$ in $\pi^{-1}(B)$ are all disjoint from each other.

For our purposes the point of this lemma is the following obvious corollary.

Corollary 2.1. For B sufficiently small there is a natural bijection between the points of $\pi^{-1}(p)$ and the connected components of $\pi^{-1}(B-D)$. The bijection is given by associating to each connected component $X$ of $\pi^{-1}(B-D)$ the intersection of the closure of $X$ in $\pi^{-1}(B)$ with $\pi^{-1}(p)$.

Next we see that exactly the same result is true if we replace $\overline{S H}_{k, b}$ with $N(\Sigma, n)$ and $\pi: \overline{S H}_{k, b} \rightarrow \mathbb{P}^{w}$ with $q: N(\Sigma, n) \rightarrow \mathbb{P}^{w}$.

Proposition 2.1. For $B$ as in Lemma 2.1 sufficiently small there is a natural bijection between the points of $q^{-1}(p)$ and the connected components of $q^{-1}(B-D)$. The bijection is given by associating to each connected component $X$ of $q^{-1}(B-D)$ the intersection of the closure of $X$ in $q^{-1}(B)$ with $q^{-1}(p)$.

Proof. The point $p$ must consist of $\ell$ distinct points $p_{1}, \ldots, p_{\ell}$ and $\ell^{\prime}$ multiple points $e_{1}, \ldots, e_{\ell^{\prime}}$ of $\mathbb{P}^{1}$. Say the multiplicity of $e_{i}$ is $k_{i}$.

Step 1. Given any connected component $X$ of $q^{-1}(B-D)$ we can find an $\left(f: \Sigma \rightarrow S^{2}\right) \in X$ such that all the $p_{i}$ 's are branch points of $f$ and none of the $e_{i}$ 's are branch points of $f$. We can then create a decorated function $\left(f, E,\left\{D_{e}\right\}_{e}\right)$ representing a point of $q^{-1}(p)$ as follows.

First, the existence of such an $f$ is clear because $q(X)=B-D$. Because $f \in X$, for small $B$ we have that if we let $D_{e_{i}}$ be a small disk around $e_{i}$, then $D_{e_{i}}$ contains exactly $k_{i}$ branch points. Letting $E=\left\{e_{1}, \ldots, e_{\ell^{\prime}}\right\},\left(f, E,\left\{D_{e_{i}}\right\}_{e_{i}}\right)$ represents a point of $q^{-1}(p)$.

Step 2. Given any point $x_{0}$ of $q^{-1}(p)$ we can choose a decorated function $\left(f, E,\left\{D_{e}\right\}_{e}\right)$ representing $x_{0}$ that is obtained in the manner of Step 1, for some connected component of $q^{-1}(B-D)$ possibly depending on $x_{0}$.

It is simply a matter of using an isotopy $\varphi_{t}$ to shrink the disks $D_{e}$ until they are small enough so that $\varphi_{t} f \in q^{-1}(B-D)$.

Step 3. Two decorated functions $\left(f, E,\left\{D_{e}\right\}_{e}\right)$ and $\left(f^{\prime}, E,\left\{D_{e}^{\prime}\right\}\right)$ obtained as in steps 1 and 2 are equivalent iff $f$ and $f^{\prime}$ lie in the same connected component of $q^{-1}(B-D)$.

By applying isotopies we can assume the disks $D_{e}=D_{e}^{\prime}$ for all $e \in E$. If $f$ and $f^{\prime}$ lie in the same connected component $X$, then a path in $X$ connecting them can be used to create the desired equivalence as in $[\mathbb{N T}]$, proof of Theorem 3.9, 
the paragraph beginning "Since the Hurwitz space $H_{g, n}$ is connected ...". On the other hand suppose $\left(f, E,\left\{D_{e}\right\}_{e}\right)$ and $\left(f^{\prime}, E,\left\{D_{e}\right\}_{e}\right)$ are equivalent. Since as points of $H(\Sigma, n) f$ and $f^{\prime}$ are well defined only up to homeomorphisms $\alpha: \Sigma \rightarrow \Sigma$ we may assume that $\left(f, E,\left\{D_{e}\right\}_{e}\right)$ and $\left(f^{\prime}, E,\left\{D_{e}\right\}_{e}\right)$ are isotopic. Let $a_{0}, a_{1} \in B-D$ correspond to the branch points of $f$ and $f^{\prime}$ respectively. Following the branch points of $f$ along the isotopy gives a path in $\mathbb{P}^{w}-D$ starting at $a_{0}$ and ending at $a_{1}$ that lifts to a path in $H(\Sigma, n)$ joining $f$ to $f^{\prime}$. Under the isotopy the branch points not in any $D_{e}$ never move and the branch points in each $D_{e}$ always stay inside disks centered at $e$. At all stages of the isotopy these disks must remain disjoint from each other and from the branch points not in any $D_{e}$. This says that the branch points in each $D_{e}$ may loop around each other, but they may not loop around branch points in other $D_{e}$ 's or branch points not in any $D_{e}$. This says that the resulting path in $\mathbb{P}^{w}-D$ joining $a_{0}$ to $a_{1}$ is homotopic to a path in $B-D$ joining $a_{0}$ to $a_{1}$ that now lifts to a path in $q^{-1}(B-D)$ joining $f$ to $f^{\prime}$ so that $f$ and $f^{\prime}$ lie in the same connected component of $q^{-1}(B-D)$.

From steps $1-3$ we see that the number of points in $q^{-1}(p)$ equals the number of connected components of $q^{-1}(B-D)$. To complete the proof it is enough to show that no connected component of $q^{-1}(B-D)$ can have more than one point of $q^{-1}(p)$ in its closure. Assume to the contrary that some connected component had at least two points of $q^{-1}(p)$ in its closure. From [NT], Lemma 2.2, we know that $N(\Sigma, n)$ is Hausdorf. We can find disjoint open neighborhoods of these two points. After possibly shrinking $B$ the intersection of $q^{-1}(B-D)$ with these two disjoint open neighborhoods would lead to two connected components, a contradiction.

We can now define the map $g$ on points of $N(\Sigma, n)$ lying over points of $D \subset \mathbb{P}^{w}$. Given $p \in D$ and $x \in q^{-1}(p)$, for a sufficiently small open ball $B$ around $p, x$ will be the only point of $q^{-1}(p)$ lying in the closure of some connected component $X$ of $q^{-1}(B-D)$. Similarly, the closure of $X$ in $\pi^{-1}(B)$ contains a unique point $y$ of $\pi^{-1}(p)$. Define $g(x)=y$.

Proposition 2.2. The map $g$ is a homeomorphism.

Proof. It is clear that $g$ is bijective. We show that $g$ is continuous. The proof that $g^{-1}$ is continuous is similar.

Let $U \subset \overline{S H}_{k, b}$ be an open set. We wish to show that $g^{-1}(U) \subset N(\Sigma, n)$ is open. For this it is sufficient to show that for each $x \in g^{-1}(U)$ we can find an open set $V_{x}$ of $N(\Sigma, n)$ with $x \in V_{x} \subset g^{-1}(U)$. Clearly this can be done for $x \in H(\Sigma, n)$ so we assume $x \in N(\Sigma, n)-H(\Sigma, n)$. Set $y=g(x)$. For a sufficiently small ball $B$ around $\pi(y)$ the connected component $Y$ of $\pi^{-1}(B)$ containing $y$ will be an open neighborhood of $y$ contained in $U$. The connected component $X$ of $q^{-1}(B)$ containing $x$ will be an open neighborhood of $x$. We wish to show $g(X) \subset Y$, so that $X \subset g^{-1}(U)$. Clearly $g(X \cap H(\Sigma, n))=Y \cap H(\Sigma, n)$, in fact $X \cap H(\Sigma, n)=Y \cap H(\Sigma, n)$. Pick any $x_{0} \in X-H(\Sigma, n)$. To compute $g\left(x_{0}\right)$ we find a sufficiently small ball $B_{0}$ around $q\left(x_{0}\right)$, we can assume $B_{0} \subset B$, so that Corollary 2.1 and Proposition 2.1 apply. Say $X_{0}$ is the connected component of $q^{-1}\left(B_{0}-D\right)$ with $x_{0}$ in its closure. Then $g\left(x_{0}\right)$ will be the point over $q\left(x_{0}\right)$ in the closure of $X_{0}$ in $\pi^{-1}\left(B_{0}\right)$. But $B_{0} \subset B$ says $X_{0} \subset X \cap H(\Sigma, n)=Y \cap H(\Sigma, n)$, so $g\left(x_{0}\right) \in Y$.

As pointed out in [DE], section $4.4, \overline{S H}_{k, b}$ is a projective variety and it is certainly normal. In view of the homeomorphism $g$ we could define a complex structure on 
$N(\Sigma, n)$ to be the corresponding complex structure on $\overline{S H}_{k, b} . N(\Sigma, n)$ would then be a normal projective variety. Since it is normal its singularities have complex codimension at least 2. As we shall see in the next section $N(\Sigma, n)$ can have singularities.

\section{A Singular EXAmple}

We shall study $N(\Sigma, n)$ when $\Sigma=S^{2}=\mathbb{P}^{1}$ and $n=3$. Thus we are studying degree three covers of $S^{2}$ simply branched at four points. We have the map $q: N\left(S^{2}, 3\right) \rightarrow \mathbb{P}^{4}$. We will show that over points of $\mathbb{P}^{4}$ corresponding to two distinct points of $\mathbb{P}^{1}$ each taken with multiplicity $2, N\left(S^{2}, 3\right)$ has two points-one nonsingular and one singular.

Let $D \subset \mathbb{P}^{4}$ be the discriminant locus consisting of nondistinct points and fix $O \in D$ where $O$ corresponds to two distinct points each with multiplicity 2 . Locally near $O, D$ consists of two smooth branches crossing transversally. Each branch corresponds to allowing one of the two multiplicity 2 points to become two distinct points. Pick a point $P \in \mathbb{P}^{4}-D$ near $O$. By standard techniques from Hurwitz space theory (see $\mathrm{F}$, proof of Proposition 1.5, $\mathrm{A}$, proof of Theorem 2.7, or [DE], section 4.2 shortly before Lemma 4.2 ) the fiber of $q$ over $P$ corresponds to equivalence classes of ordered 4 -tuples of simple transpositions $\left[\sigma_{1}, \sigma_{2}, \sigma_{3}, \sigma_{4}\right], \sigma_{i} \in S_{3}$ (the symmetric group on three letters), such that the product $\sigma_{1} \sigma_{2} \sigma_{3} \sigma_{4}=(1)$ and $\left\{\sigma_{1}, \sigma_{2}, \sigma_{3}, \sigma_{4}\right\}$ generates a transitive subgroup of $S_{3}$, where the equivalence relation is $\left[\sigma_{1}, \sigma_{2}, \sigma_{3}, \sigma_{4}\right]$ is equivalent to $\left[\tau_{1}, \tau_{2}, \tau_{3}, \tau_{4}\right]$ iff there exists an $\alpha \in S_{3}$ such that $\sigma_{i}=\alpha \tau_{i} \alpha^{-1}, i=1, \ldots, 4$. Each $\sigma_{i}$ represents the ramification point over one of the four points of $\mathbb{P}^{1}$ represented by $P$. One computes that over $P$ there are four points which we may represent as: [(12), (12), (23), (23)], [(12), (23), (12), (13)], $[(12),(23),(13),(23)]$, and $[(12),(23),(23),(12)]$.

We may assume that we have set things up so that one branch of $D$ near $O$ corresponds to the first two points becoming one double point and the other branch represents the last two points becoming one double point. Again from standard Hurwitz space techniques (see $[\mathrm{F}]$, proof of Proposition 1.5, [A], proof of Theorem 2.7, or $[\mathrm{DE}$, end of section 4.2) we see that the monodromy action on the inverse image of $P$ generated by a loop based at $P$ going around branch 1 is generated by $\left[\sigma_{1}, \sigma_{2}, \sigma_{3}, \sigma_{4}\right] \mapsto\left[\sigma_{2}, \sigma_{2}^{-1} \sigma_{1} \sigma_{2}, \sigma_{3}, \sigma_{4}\right]$ and around branch 2 it is $\left[\sigma_{1}, \sigma_{2}, \sigma_{3}, \sigma_{4}\right] \mapsto\left[\sigma_{1}, \sigma_{2}, \sigma_{4}, \sigma_{4}^{-1} \sigma_{3} \sigma_{4}\right]$. One computes that in both cases $[(12),(12),(23),(23)]$ does not move but that the other three points are permuted cyclically. Remember that after applying the monodromy transformation you might need to conjugate by an appropriate element of $S_{3}$ to get the ordered 4-tuple to be one of the four we have chosen to represent the fiber.

Thus over a small neighborhood of $O$ in $\mathbb{P}^{w}, N\left(S^{2}, 3\right)$ has two components. One is a single sheet mapping isomorphically onto the small neighborhood. This gives the nonsingular point of $q^{-1}(O)$. The other component consists of three sheets all coming together and ramifying to order 3 over each branch of $D$. We now concentrate on that component; call it $X$.

Choose local coordinates $u, v, x, y$ on $\mathbb{P}^{4}$ near $O$ so that $D$ has local equation $x y=0$. The ramification to order 3 along both $x=0$ and $y=0$ says that in $X, x y$ has a cube root. In $\mathbb{C}^{5}$ with coordinates $u, v, x, y, z$ take the hypersurface $X^{\prime}=\left\{x y=z^{3}\right\} . \quad X^{\prime}$ maps to $\mathbb{P}^{4}$ by $(u, v, x, y, z) \mapsto(u, v, x, y)$ (locally near $O$ of course). One easily computes that the singularities of $X^{\prime}$ are $x=y=z=0$. 
$X^{\prime}$ is normal because it is a hypersurface with singularities in codimension greater than 1; see $\mathrm{Ha}$, Proposition II.8.23. $X^{\prime}$ also has the appropriate monodromy along $x y=0$. By uniqueness of normalization $X^{\prime}$ near $(0, \ldots, 0)$ is isomorphic to $X$ near $q^{-1}(O) \cap X$. Thus $X$ is singular.

Even if we get a loop backwards in the monodromy the only other possibility is $z^{3}=x^{2} y$ which also has a singular normalization.

As a final remark we note that since $\overline{S H}_{k, b}$ is normal any nonsingular variety $Z$ finite over $\mathbb{P}^{w}$ compactifying $H^{n, w}$ would be isomorphic to $\overline{S H}_{k, b}$. Since a nonsingular variety is normal such a $Z$ would have to be the normalization of $\mathbb{P}^{w}$ in the function field of $H^{n, w}$, hence equal to $\overline{S H}_{k, b}$. Thus we cannot make $N\left(S^{2}, 3\right)$ nonsingular by finding a different complex structure to put on it.

\section{REFERENCES}

[A] E. Arbarello, On subvarieties of the moduli space of curves of genus g defined in terms of Weierstrass points, Lincei-Mem. Sci. Fis., ecc.-1978-S. VIII, Vol. XV, Sez. I, 1. MR 80f:14013

[DE] S. Diaz and D. Edidin, Towards the Homology of Hurwitz Spaces, J. Differential Geometry Vol. 43, No. 1, (1996), 66-98. MR 98e:14028

[F] W. Fulton, Hurwitz schemes and the irreducibility of moduli of algebraic curves, Ann. Math. 90 (1969), 542-575. MR 41:5375

[Ha] R. Hartshorne, Algebraic Geometry, Springer-Verlag, New York, 1977. MR 57:3116

$[\mathrm{HM}] \mathrm{J}$. Harris and D. Mumford, On the Kodaira dimension of the moduli space of curves, Inventiones Mathematicae 67 (1982), 23-86. MR 83i:14018

[Hu] A. Hurwitz, Über Riemann'sche Flächen mit gegeberen Verzweigungspunkten, Math. Ann. 39 (1891), 1-61.

[M] S. Mochizuki, The geometry of the compactification of the Hurwitz scheme, Publications RIMS, Kyoto University, 31 (1995), 355-441. MR 96j:14017

[NT] S. Natanzon and V. Turaev, A compactification of the Hurwitz space, Topology 38, No. 4, (1999), 889-914. MR 2000b:57004

Department of Mathematics, Syracuse University, Syracuse, New York 13244

E-mail address: spdiaz@mailbox.syr.edu 